\title{
Desenvolvimento do omaso de bovinos da raça Nelore (Bos taurus indicus) durante o período pré-natal
}

\author{
Development of the omasum of Nelore cattle (Bos taurus indicus) during the prenatal \\ period
}

\author{
Gabriela Mansano do NASCIMENTO' ${ }^{1}$; Silvia Helena Venturoli PERRI ${ }^{2}$; Roberto Gameiro de \\ CARVALHO $^{2}$; Cristina Maria Rodrigues MONTEIRO ${ }^{2}$
}

\author{
${ }^{1}$ Graduanda em Medicina Veterinária, Bolsista FAPESP. \\ ${ }^{2}$ Departamento de Apoio, Produção e Saúde Animal da Faculdade de Medicina Veterinária da Universidade Estadual Paulista, \\ Araçatuba - SP, Brasil.
}

\begin{abstract}
Resumo
O desenvolvimento do estômago de mamíferos domésticos é um processo complexo, especialmente em ruminantes. Essa pesquisa analisou o desenvolvimento desse órgão durante o período pré-natal. Foram utilizadas amostras de omaso de fetos da raça Nelore (Bos taurus indicus) divididos em cinco grupos com seis amostras cada: $1^{\circ}$ - fetos com 9 a 15 semanas (8 a $21 \mathrm{~cm}$ ) de gestação, $2^{\circ}$ - fetos com 16 a 22 semanas $(23$ a $37 \mathrm{~cm}), 3^{\circ}-$ fetos com 23 a 29 semanas $(40$ a $58 \mathrm{~cm}), 4^{\circ}-$ fetos com 30 a 36 semanas $\left(61\right.$ a $77 \mathrm{~cm}$ ) e $5^{\circ}-$ fetos com 37 a 43 semanas $(79$ a $88 \mathrm{~cm}$ ). Os cortes histológicos foram corados com Hematoxilina e Eosina e Picrossirius e examinados ao microscópio de luz. No feto de $11 \mathrm{~cm}$, foi observado mucosa com lâminas primárias, secundárias e terciárias uniformes e demais camadas presentes. No feto de $13,5 \mathrm{~cm}$ surgiram as lâminas quaternárias, papilas nas lâminas primárias e secundárias e a formação da muscular da mucosa. Aos $28 \mathrm{~cm}$, as papilas estavam em pleno desenvolvimento, assim como as lâminas, principalmente as quaternárias. No feto de $42 \mathrm{~cm}$ houve início de queratinização epitelial e, no de $56 \mathrm{~cm}$, estava totalmente queratinizado. No feto de $60 \mathrm{~cm}$, as lâminas possuíam papilas grandes e afiladas no ápice e, aos $65 \mathrm{~cm}$, houve diminuição do epitélio e aumento da camada muscular. $\mathrm{O}$ feto de $83 \mathrm{~cm}$ apresentou lâminas delgadas, muscular da mucosa discreta e papilas por toda a superfície. Concluiu-se que as lâminas e papilas foram as estruturas que mais apresentaram modificações durante o desenvolvimento fetal.
\end{abstract}

Palavras-chave: Morfologia. Ventrículo Pluricavitário. Fetos. Ruminantes.

\begin{abstract}
The development of the stomach of domestic mammals is a complex process, especially in ruminants. The aim of this study was to analyze this organ during prenatal period. Omasum samples of Nelore fetuses (Bos taurus indicus) were divided into five groups of six samples each: 1 - fetuses from 9 to 15 weeks ( 8 to $21 \mathrm{~cm}$ ) of gestation, 2 - fetuses from 16 to 22 weeks ( 23 to $37 \mathrm{~cm}$ ), 3 - fetuses from 23 to 29 weeks ( 40 to $58 \mathrm{~cm}$ ), 4 - fetuses from 30 to 36 weeks $(61$ to $77 \mathrm{~cm}$ ) and 5 - fetuses from 37 to 43 weeks ( 79 to $88 \mathrm{~cm}$ ). The histological sections were stained with Hematoxylin and Eosin and Picrossirius and examined by light microscopy. In fetuses of $11 \mathrm{~cm}$ it was observed mucosa with uniform primary, secondary and tertiary blades and all layers present. The fetus with $13.5 \mathrm{~cm}$ presented quaternary blades emergence, papillae on primary and secondary blades and formation of the muscularis mucosae. At $28 \mathrm{~cm}$ it were observed papillae in development, as well as the blades, especially the quaternary. In $42 \mathrm{~cm}$ fetuses was early epithelial keratinization and at $56 \mathrm{~cm}$ it was fully keratinized. In $60 \mathrm{~cm}$ fetuses the blades presented large papillae with tapered apex and at $65 \mathrm{~cm}$ there was decreased epithelium and increase of the muscle layer. In the fetus of $83 \mathrm{~cm}$ it were observed thin sections, discrete muscularis mucosa and papillae over the entire organ surface. It was concluded that the blades and papillae were structures that presented more changes during fetal development.
\end{abstract}

Keywords: Morphology. Pluricavitary Ventricle. Fetuses. Ruminants.

\section{Introdução}

É notável a habilidade dos ruminantes para converter fibras em produtos de grande valor nutritivo, por isso, o conhecimento científico na estrutura e função do trato digestório desses animais é de grande interesse ${ }^{1}$.

O desenvolvimento do estômago dos mamíferos domésticos é um processo complexo ${ }^{2}$. Especial-

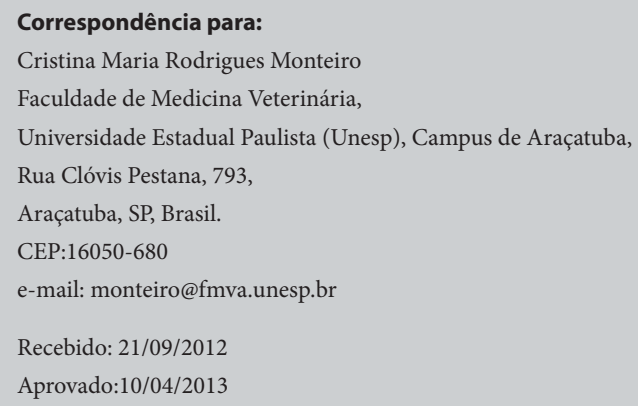


mente em ruminantes, o desenvolvimento do estômago composto cria um grande desafio para a embriologia ${ }^{3,4,5,6}$.

A rotação do estômago e outros processos de desenvolvimento que ocorrem na vida pré-natal já são bem conhecidos $^{7,8}$. Contudo, Franco et al. ${ }^{5}$ destacaram a ausência de estudos mais detalhados e de informações mais amplas a respeito da morfologia e desenvolvimento do estômago de bovinos no período pré-natal. Deste modo o presente trabalho investigou o desenvolvimento do omaso empregando análises histológicas e, sobretudo, histomorfométricas de modo a oferecer dados numéricos que complementam os conhecimentos sobre esse assunto, principalmente no que tange aos zebuínos, uma vez que estes animais são largamente difundidos no país assumindo grande importância econômica.

\section{Material e Método}

Foram utilizadas amostras de omaso de fetos bovinos da raça Nelore (Bos taurus indicus) coletadas em frigorífico localizado no município de Birigui, estado de São Paulo, separados em cinco grupos: $1^{\circ}$ grupo - fetos com aproximadamente 9 a 15 semanas (8 a $21 \mathrm{~cm}$ ) de gestação, $2^{\circ}$ grupo - fetos com 16 a 22 semanas $(23$ a $37 \mathrm{~cm}), 3^{\circ}$ grupo - fetos com 23 a 29 semanas $(40 \mathrm{a} 58 \mathrm{~cm}), 4^{\circ}$ grupo - fetos com 30 a 36 semanas (61 a $77 \mathrm{~cm})$ e $5^{\circ}$ grupo - fetos com 37 a 43 semanas (79 a $88 \mathrm{~cm})$. As idades dos fetos foram estabelecidas com o emprego da tabela de Baraldi e Zietzschamm-Krolling (mensuração da nuca até a transição sacrococcígea). Para as análises histológica e histomorfométrica, fragmentos coletados dos órgãos foram fixados em solução tamponada de paraformaldeido a 5\%, durante 24 horas para processamento com Paraplast ${ }^{\oplus}$ e se proceder a microtomia. Os cortes histológicos de aproximadamente $5 \mu \mathrm{m}$ de espessura foram corados com Hematoxilina e Eosina e Picrossirius As estruturas histológicas foram analisadas por período de gestação e a histomorfometria efetuou mensurações $(\mu \mathrm{m})$ da parede total, do epitélio, lâmina própria e submucosa, muscular (subcamadas interna e externa) e serosa, utilizando-se o programa de análises de imagens Image J. Os resultados obtidos foram submetidos à análise de variância comparando-se os valores médios com o teste de Tukey. O nível de significância adotado foi de 0,05 . As análises estatísticas foram efetuadas com o emprego do programa computacional SAS (Statistical Analysis System) versão 8.2.

\section{Resultados Histológicos}

Grupo 1 - fetos com 9 a 15 semanas de gestação $(8$ a $21 \mathrm{~cm})$.

$\mathrm{O}$ omaso do feto com $11 \mathrm{~cm}$ apresentou mucosa com lâminas primárias, secundárias e terciárias uniformes, delgadas e ovaladas revestidas no seu ápice por epitélio estratificado com aproximadamente sete fileiras de células, cuja porção basal foi constituída por dois estratos celulares apresentando condensação nuclear e as demais células com citoplasma ovalado e claro com núcleo deslocado. A lâmina própria formada de tecido conjuntivo frouxo com células difusas e centralmente apresentou três camadas de mioblastos originados da camada muscular interna. A submucosa, pouco visível, formada por tecido frouxo permaneceu com esta morfologia até o final. A camada muscular apresentou-se dividida em duas subcamadas, a interna circular espessa e a longitudinal externa mais delgada. A camada serosa apresentou-se delgada com algumas áreas espessas constituída de tecido conjuntivo e mesotélio externamente (Figura 1A). No feto de $13,5 \mathrm{~cm}$, algumas lâminas mostraram-se irregulares e outras com ápice dilatado e em aspecto de raquete. Também foram constatadas lâminas quaternárias e o início da formação das papilas nas lâminas de primeira e de segunda ordem. Observou-se nessa fase o início da formação da muscular da mucosa (Figura 1B). Aos 15 e 16,5 cm não foram registradas modificações evidentes e com $17,5 \mathrm{~cm}$, observou-se peque- 

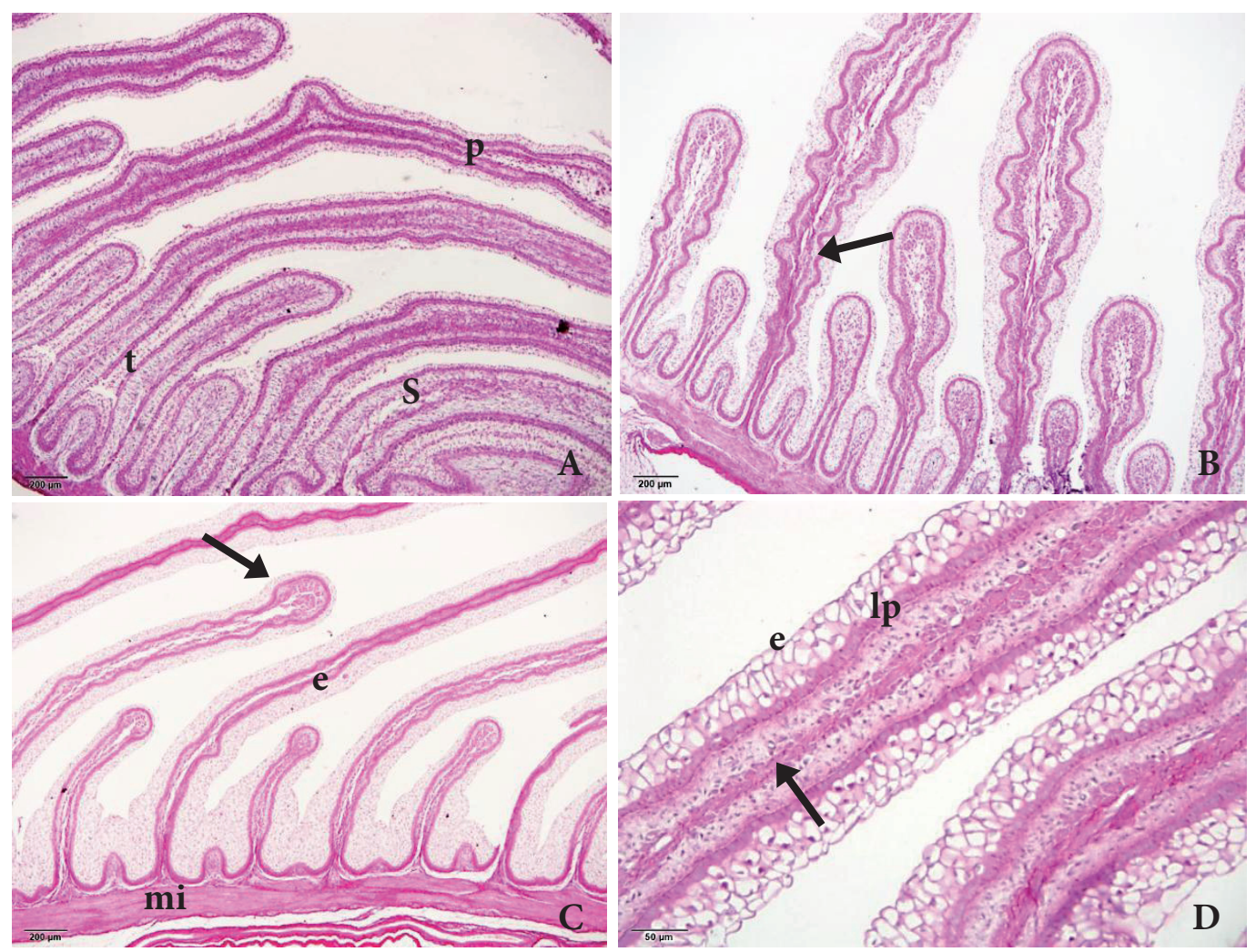

Figura 1 - Fotomicrografia de omaso de Bos taurus indicus. A - feto com $11 \mathrm{~cm}$. Grupo 1. Lâmina primária (p). Lâmina secundária (s). Lâmina terciária (t). Obj. 4 . B - feto com $13,5 \mathrm{~cm}$. Grupo 1. Lâminas irregulares (seta). Muscular da mucosa delgada (cabeça de seta). Obj. 4. C - feto com $28 \mathrm{~cm}$. Grupo 2. Ápice da lâmina com aspecto de raquete (seta). Epitélio estratificado (e). Muscular interna (mi). Obj. 4 . D - feto com $31 \mathrm{~cm}$. Grupo 2. Epitélio estratificado (e). Lâmina própria (lp). Muscular da mucosa evidente (mm). Obj. 20. Picrossirius - Araçatuba - 2012

no aumento do epitélio. Com $18,5 \mathrm{~cm}$, as papilas já estavam bem desenvolvidas e as camadas muscular e serosa eram espessas.

Grupo 2 - fetos com 16 a 22 semanas de gestação $(23$ a $37 \mathrm{~cm})$.

No feto de $26 \mathrm{~cm}$ foi observada a mucosa com papilas bem desenvolvidas presentes em toda a superfície das lâminas primárias e secundárias, camada muscular da mucosa delgada. A submucosa continuou pouco distinguível, porém, as camadas muscular e serosa eram espessas. Aos $28 \mathrm{~cm}$ as papilas apresentaram-se em pleno desenvolvimento, assim como as lâminas, principalmente as quaternárias, mantendo o ápice em forma de raquete, porém, a muscular da mucosa permaneceu delgada (Figura $1 \mathrm{C}$ ). Com $29 \mathrm{~cm}$ o epitélio apresentou-se espessado com aproximadamente 11 estratos celulares, muscular da mucosa delgada, em desenvolvimento contínuo e a camada serosa era típica. $\mathrm{O}$ feto de $31 \mathrm{~cm}$ apresentou a mesma morfologia anterior, porém a muscular da mucosa era bem desenvolvida (Figura 1 D). No feto de $33,5 \mathrm{~cm}$ as camadas eram semelhantes às do feto anterior, porém a camada muscular apresentou aumento em sua espessura mantendo o padrão morfológico: subcamada interna mais espessa que a externa. Com $37 \mathrm{~cm}$ observou-se mucosa com lâminas primárias revestidas por epitélio alto em aproximadamente 20 estratos celulares, presença de papilas nas lâminas 
terciárias e camada muscular mais espessa com delimitação clara entre as subcamadas.

Grupo 3 - fetos com 23 a 29 semanas de gestação (40 a $58 \mathrm{~cm})$.

No feto de $42 \mathrm{~cm}$, o omaso exibiu lâminas delgadas com papilas desenvolvidas revestidas por epitélio constituído por aproximadamente 15 estratos celulares com citoplasma ovalado e núcleos excêntricos, as células mais superficiais apresentaram-se com formato arredondado e núcleos centrais com início de queratinização. A muscular da mucosa era delgada. A camada muscular mostrou-se mais espessa e a serosa delgada (Figura $2 \mathrm{~A}$ ). Com $43 \mathrm{~cm}$, a mucosa apresentou lâminas quaternárias com papilas. $\mathrm{O}$ epitélio de revestimento era formado por 18 estratos de células menores, arredondadas, queratinizadas e com núcleos centrais. A muscular da mucosa era bem evidente. Aos $44 \mathrm{~cm}$, as lâminas eram delgadas com papilas bem desenvolvidas revestidas por epitélio irregular. A camada muscular e a serosa eram mais espessas. No feto de $48 \mathrm{~cm}$ foi visualizada a mesma morfologia que o anterior, porém, a camada muscular era bem mais espessa, assim como no feto de $52 \mathrm{~cm}$. Com $56 \mathrm{~cm}$, as lâminas eram mais espessas com papilas bem evidentes revestidas por epitélio totalmente queratinizado.

Grupo 4 - fetos com 30 a 36 semanas de gestação (61 $\mathrm{a} 77 \mathrm{~cm})$.

No feto de $60 \mathrm{~cm}$ o omaso apresentou lâminas com papilas grandes e afiladas no seu ápice. Aos $65 \mathrm{~cm}$ o epitélio apresentou redução para 10 estratos e estava altamente queratinizado com células superficiais pavimentosas e muscular da mucosa bem espessa (Figura $2 \mathrm{~B})$. Houve constante desenvolvimento das túnicas em relação aos achados anteriores, porém, o que se verificou com mais determinação foi o aumento em espessura da camada muscular, fato observado no feto de $66 \mathrm{~cm}$. Os omasos de fetos com $68 \mathrm{~cm}$ e $70 \mathrm{~cm}$ apresentaram morfologias semelhantes: epitélio com condensação nuclear na superfície basal, presença de lâminas delgadas com papilas grandes e arredondadas, lâmina própria com células difusas e muscular da mucosa bem desenvolvida. A submucosa continuou pouco distinguível, camada muscular bem desenvolvida e serosa delgada. Aos $75 \mathrm{~cm}$ foi constatado aumento no tamanho das papilas localizadas na porção apical das lâminas.

Grupo 5 - fetos com 37 a 43 semanas de gestação (79 a $88 \mathrm{~cm})$.

No feto de $81 \mathrm{~cm}$ o omaso apresentou epitélio estratificado queratinizado com aproximadamente 15 estratos de células, lâminas espessas com muscular da mucosa bem saliente na porção apical. Papilas grandes arredondas e afiladas estavam presentes por toda a superfície das lâminas. O feto de $83 \mathrm{~cm}$ apresentou-se distinto do anterior, lâminas delgadas com ápice pouco dilatado, muscular da mucosa discreta e papilas arredondadas e afiladas por toda a superfície. A camada muscular era espessa e a serosa ora delgada ora espessa constituída por tecido conjuntivo e mesotélio (Figura $2 \mathrm{C}$ e D).

\section{Resultados Histomorfométricos}

$\mathrm{Na}$ tabela 1, pode-se verificar que os valores da camada muscular e da parede total foram crescentes para todos os grupos, porém, houve diferenças significantes entre eles. As médias das espessuras do epitélio, lâmina própria e submucosa e serosa não apresentaram diferença significante entre os grupos. $\mathrm{Na}$ camada muscular não foi observada diferença significativa entre os grupos 3, 4 e 5, mas houve diferença do grupo 5 em relação aos grupos 1 e 2 que também não apresentaram diferença entre si. Para a camada serosa não houve diferença significante entre os grupos. Para a parede total não houve diferença significativa entre os grupos 2, 3, 4 e 5, mas houve diferença dos grupos 4 e 5 em relação ao grupo 1 . 

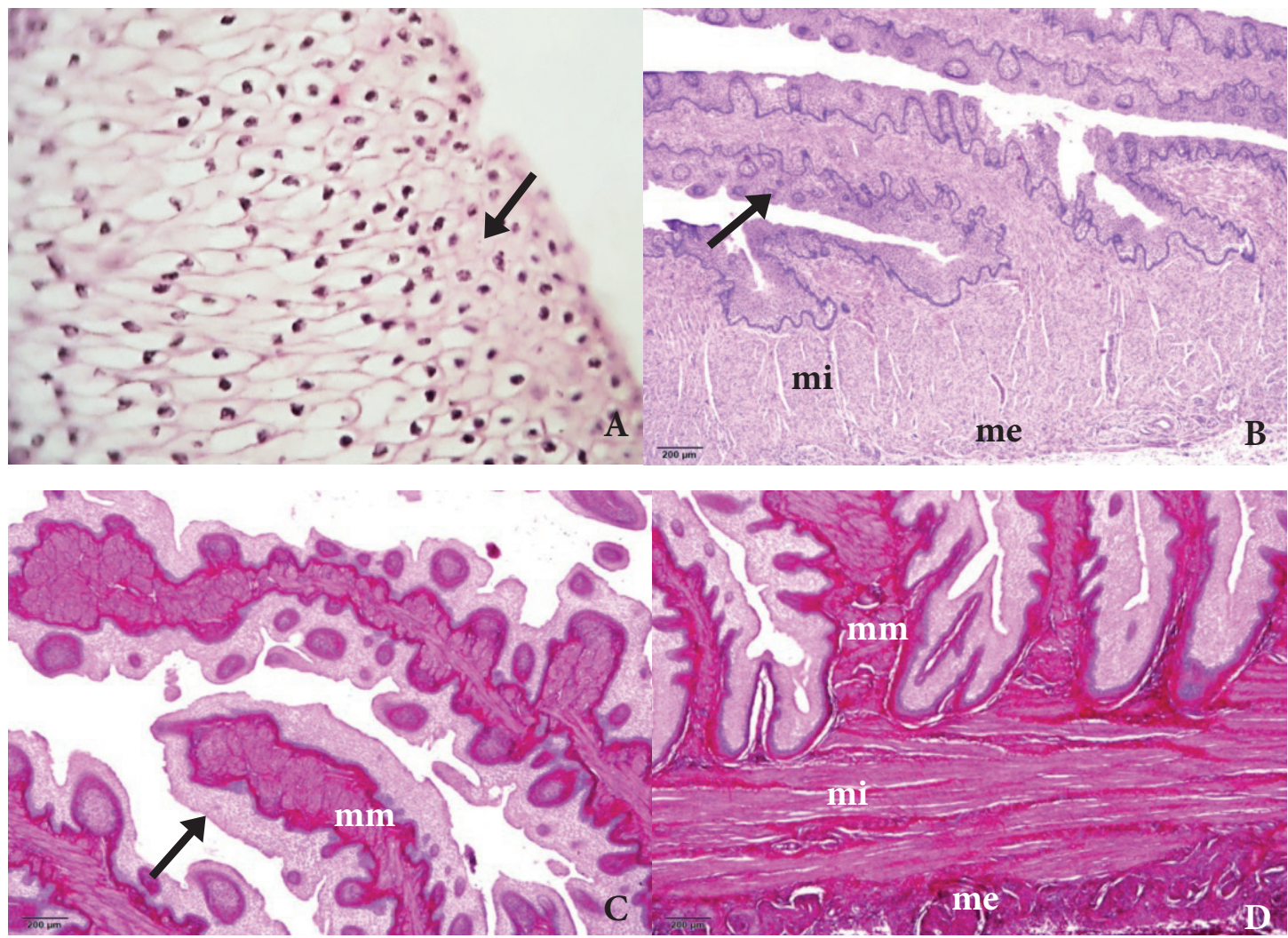

Figura 2 - Fotomicrografia de omaso de Bos taurus indicus. A - feto com $42 \mathrm{~cm}$. Grupo 3. Células no inicio de queratinização na porção superior do epitélio (seta). Obj. 40 . B - feto com $65 \mathrm{~cm}$. Grupo 4. Lâmina com epitélio queratinizado (seta). Muscular interna (mi). Muscular externa (me). Obj. 4. Hematoxilina e Eosina. C - feto com $83 \mathrm{~cm}$. Grupo 5. Epitélio (seta). Muscular da mucosa (mm). Obj. 4. D - feto com $83 \mathrm{~cm}$. Grupo 5. Muscular da mucosa (mm). Muscular interna (mi). Muscular externa (me). Obj. 4. Picrossirius - Araçatuba - 2012

Tabela 1 - Média $(\overline{\mathrm{X}})$ e desvio padrão $(\mathrm{S})$ das espessuras, em $\mu \mathrm{m}$, do epitélio, lâmina própria+submucosa $(\mathrm{Lp}+\mathrm{Sb})$, muscular, serosa e parede total do omaso de Bos taurus indicus - Araçatuba (SP) - 2012

\begin{tabular}{cccccc}
\hline \multirow{2}{*}{ Variável } & \multicolumn{5}{c}{ Espessura $(\overline{\mathrm{X}} \pm \mathrm{S})$} \\
\cline { 2 - 5 } & $\mathrm{G} 1$ & $\mathrm{G} 2$ & $\mathrm{G} 3$ & $\mathrm{G} 4$ & $\mathrm{G} 5$ \\
\hline Epitélio & $102 \pm 22^{\mathrm{a}}$ & $164 \pm 49^{\mathrm{a}}$ & $165 \pm 43^{\mathrm{a}}$ & $174 \pm 71^{\mathrm{a}}$ & $121 \pm 4^{\mathrm{a}}$ \\
Lp+Sb & $44 \pm 12^{\mathrm{a}}$ & $51 \pm 6^{\mathrm{a}}$ & $56 \pm 22^{\mathrm{a}}$ & $64 \pm 30^{\mathrm{a}}$ & $59 \pm 16^{\mathrm{a}}$ \\
Muscular & $104 \pm 16^{\mathrm{c}}$ & $252 \pm 78^{\mathrm{bc}}$ & $363 \pm 156^{\mathrm{abc}}$ & $528 \pm 210^{\mathrm{ab}}$ & $632 \pm 331^{\mathrm{a}}$ \\
Serosa & $32 \pm 8^{\mathrm{a}}$ & $26 \pm 2^{\mathrm{a}}$ & $26 \pm 2^{\mathrm{a}}$ & $27 \pm 9^{\mathrm{a}}$ & $25 \pm 1^{\mathrm{a}}$ \\
Parede total & $283 \pm 38^{\mathrm{b}}$ & $493 \pm 124^{\mathrm{ab}}$ & $610 \pm 203^{\mathrm{ab}}$ & $793 \pm 270^{\mathrm{a}}$ & $837 \pm 344^{\mathrm{a}}$ \\
\hline
\end{tabular}

Médias com letras distintas na mesma linha diferem entre si pelo teste de Tukey $(\mathrm{P}<0,05)$ 


\section{Discussão e Conclusões}

Os omaso dos fetos com $11 \mathrm{~cm}$ apresentaram a mucosa constituída por lâminas primárias, secundárias e terciárias o que difere da constatação de Franco et al. ${ }^{9}$ que em fetos de ovelha registraram o aparecimento das lâminas em diferentes períodos e também difere das observações de Masot, Franco e Redondo ${ }^{10}$ e Redondo, Franco e Masot ${ }^{11}$ que observaram a mesma ocorrência em cabras selvagens o que sugere que nos pequenos ruminantes as lâminas não devem aparecer logo no início da gestação como ocorre nos bovinos. Os fetos de $11 \mathrm{~cm}$ apresentaram epitélio com sete camadas de células, lâmina própria com três camadas de mioblastos centrais que provavelmente originam a muscular da mucosa, submucosa pouco desenvolvida, muscular interna e externa e serosa. Vivo et al. $^{2}$ encontraram as mesmas características, porém, em fetos com $7,8 \mathrm{~cm}$, que apresentaram apenas uma camada de mioblastos na lâmina própria, sinal inicial do desenvolvimento da muscular da mucosa. Com $13,5 \mathrm{~cm}$ houve o aparecimento das lâminas quaternárias, início da formação de papilas nas lâminas primárias e secundárias e desenvolvimento da muscular da mucosa. Vivo et al. ${ }^{2}$ registraram o mesmo perfil em fetos mais jovens, com $11,5 \mathrm{~cm}$ e Totzauer e Sinowatz ${ }^{12}$ afirmaram que as estruturas características do omaso já se faziam presentes em fetos bovinos com três meses de gestação, observações que discorda do observado no presente trabalho, onde a muscular da mucosa esteve presente em todas as lâminas no período compreendido entre quatro e cinco meses de gestação, variação esta que poderia ser atribuída à diferença entre as raças. Após este período, observaram-se apenas aumentos em espessura de algumas estruturas como epitélio e túnicas muscular e serosa.

O omaso dos fetos com $26 \mathrm{~cm}$ apresentou mucosa com papilas bem desenvolvidas nas lâminas primárias e secundárias e aos $29 \mathrm{~cm}$ foi constatado aumento em espessura do epitélio com aproximadamente 11 estra- tos celulares o que passou para 20 estratos em fetos aos $37 \mathrm{~cm}$, demonstrando portanto que na metade do período gestacional houve o aumento da espessura do epitélio com leve declínio no final. Aos $31 \mathrm{~cm}$, a muscular da mucosa tornou-se bem evidente o que concorda com os resultados de Vivo et al. ${ }^{2}$ que fizeram a mesma observação em fetos bovinos com 4,5 meses de gestação apesar desses últimos serem de raças diferentes da utilizada no presente trabalho. O omaso dos fetos com $42 \mathrm{~cm}$ apresentou início de queratinização epitelial e aos $43 \mathrm{~cm}$ todas as suas lâminas estavam recobertas por papilas o que discorda do registro de Redondo, Franco e Masot ${ }^{11}$ que verificaram que em fetos de cabras selvagens a presença das papilas em todas as lâminas foi verificada somente ao final da gestação o que confirma as variações esperadas principalmente quando são realizadas comparações entre o omasso de grandes e pequenos ruminantes. Nos fetos aos 56 $\mathrm{cm}$, foram observadas papilas bem evidentes e lâminas mais espessas recobertas por epitélio totalmente queratinizado. Vivo et $\mathrm{al}^{2}{ }^{2}$ observaram a presença de papilas por toda mucosa, porém, com a presença de queratinização epitelial apenas em fetos com $62 \mathrm{~cm}$, período mais tardio que o observado na presente pesquisa, o que provavelmente pode ser atribuído à diferenças raciais.

O omaso dos fetos com $60 \mathrm{~cm}$ apresentou papilas bem desenvolvidas com ápices afilados e posteriormente mais arredondados nos fetos com $70 \mathrm{~cm}$. A espessura do epitélio passou a apresentar 10 estratos celulares altamente queratinizados com células superficiais pavimentosas. A partir desse período houve constante desenvolvimento das camadas, sobretudo da muscular. Franco et al. ${ }^{9}$ também afirmaram que, na fase final de gestação em fetos ovinos, não houve alterações substanciais em relação aos anteriores, fato indicativo de que as principais variações morfológicas devem ocorrer do inicio até a metade da gestação. $\mathrm{O}$ omaso dos fetos com $81 \mathrm{~cm}$ e $83 \mathrm{~cm}$ apresentou lâminas espessas com papilas arredondadas e afiladas por 
toda a sua superfície recobertas por epitélio queratinizado com 15 estratos celulares e camada muscular da mucosa bem diferenciada.

Os valores da camada muscular e da parede total do omaso foram crescentes para todos os grupos com diferenças significantes entre si (Tabela 1) o que é esperado, pois à medida que o feto evolui obviamente há aumento das mensurações, fato confirmado no presente trabalho a despeito do registro de um leve declínio no final. Para as demais estruturas do epitélio, lâmina própria e submucosa e serosa não houve crescimento contínuo e diferença significativa. Vivo et al. ${ }^{2}$ constataram que após os 95 dias de gestação os fetos apresentam crescimento contínuo da camada muscular e para as demais camadas, as variações observadas nas respectivas mensurações concordam com os resultados obtidos

\section{Referências}

1. FRANCO, A. J.; REGODÓN, S.; ROBINA, A.; REDONDO, E. Histomorphometric analysis of the rumen of sheep during development. American Journal of Veterinary Research, v. 53, n. 7, p. 1209-1217, 1992.

2. VIVO, J. M.; ROBINA, A.; REGODÓN, S.; GUILLÉN, M. T.; FRANCO, A.; MAYORAL, A. I. Histogenetic evolution of bovine gastric compartments during the prenatal period. Histology and Histopathology, v. 5, n. 4, p. 461-476, 1990.

3. KNOSPE, C. Die Entwicklung der Magendruesen der Katze (Felis silvestris catus). Anatomia, Histologia, Embryologia, v. 25 , n. 2, p. 75-94, 1996.

4. SANGLID, P. T.; SCHMIDT, M.; ELNIF, J.; BJÖRNVAD, C. R.; WESTRÖM, B. R.; BUDDINGTON, R. K. Prenatal development of gastrointestinal function in the pig and the effects of fetal esophageal obstruction. Pediatric Research, v. 52, p. 416-424, 2002.

5. FRANCO, A. J.; MASOT, A. J.; AGUADO, M.C.; GÓMEZ, L.; REDONDO, E. Morphometric and immunohistochemical study of the rumen of red deer during prenatal development. Journal of Anatomy, v. 204, p. 501-513, 2004.

6. FRANCO, A.J.; REDONDO, E.; MASOT, A.J. Morphometric and immunohistochemical study of the reticulum of red deer during prenatal development. Journal of Anatomy, v. 205, p. 277-289, 2004. no presente trabalho. Redondo, Franco e Masot ${ }^{11}$ estudando a evolução do omaso de fetos de caprinos selvagens constataram o crescimento contínuo de epitélio, túnica muscular e parede total, porém a serosa, apresentou queda nas mensurações, o que difere do observado no presente trabalho no relativo aos valores do epitélio e túnica serosa e confirma que variações morfológicas no desenvolvimento de certas estruturas nem sempre apresentam um sentido crescente.

Diante dos resultados encontrados, pode-se concluir que: os valores da camada muscular e parede total do omaso foram crescentes para todos os grupos; não houve padrão contínuo de crescimento para as demais estruturas e as lâminas e papilas foram as estruturas que sofreram maiores variações durante o desenvolvimento fetal.

7. AMASAKI, H.; DAIGO, M. Morphogenesis of the epithelium and the lamina propria of the rumen in bovine fetuses and neonates. Anatomia, Histologia, Embryologia, v. 17, n. 1, p. 1-6, 2007.

8. CHRÓSZCZ, A. The morphometric and topographic study of pig's stomach development between the $35^{\text {th }}$ and $1145^{\text {th }}$ day of gestation. Electronic Journal of Polish Agricultural Universities, v. 11, n. 4, 2008.

9. FRANCO, A.; ROBINA, A.; REGODÓN, S.; VIVO, J. M.; MASOT, A. J.; REDONDO, E. Histomorphometric analysis of the omasum of sheep during development. American Journal of Veterinary Research, v. 54, n. 8, p. 1221-1229, 1993.

10.MASOT, A. J.; FRANCO, A. J.; REDONDO, E. Comparative analysis of the forestomach mucosa in red deer during prenatal development. Revue Médecine Vétérinaire, v. 158, n. 7, p. 397-409, 2007.

11.REDONDO, E.; FRANCO, A. J.; MASOT, A. J. Morphometric and immunohistochemical study of thr omasum of red deer during prenatal development. Journal of Anatomy, v. 206, n. 6 , p. $543-555,2005$.

12.TOTZAUER, I.; SINOWATZ, F. Fetal development of the omasum of cattle (Bos taurus), Tierarztliche Praxis, v. 18, n. 6 , p. $577-583,1990$. 